\title{
ACTITUDES DE LOS PROFESORES ANTE LA INTEGRACIÓN DE LAS TIC EN LA PRÁCTICA DOCENTE. ESTUDIO DE UN GRUPO DE LA UNIVERSIDAD DE VALLADOLID
}

\author{
UNIVERSITY TEACHERS' ATTITUDES TOWARDS THE EDUCATIONAL \\ USE OF ICTS: THE CASE STUDY AT THE UNIVERSITY OF VALLADOLID
}

\author{
Susana Álvarez; susanalv@lesp.uva.es \\ Carmen Cuéllar; carmen.cuellar@lesp.uva.es \\ Belén López; belenl@lia.uva.es \\ Cristina Adrada; cadrada@ffr.uva.es \\ Rocío Anguiano; anguiano@ffr.uva.es \\ Antonio Bueno; bueno@ffr.uva.es \\ Isabel Comas; isacomas@fing.uva.es \\ Susana Gómez; susanag@fing.uva.es \\ Universidad de Valladolid (España)
}

\section{RESUMEN}

Debido al proceso de adaptación y convergencia en el que nos hallamos inmersos en la actualidad y a la incorporación de los nuevos planes europeos de enseñanzas universitarias, resulta necesario plantear la inclusión de metodologías activas en el proceso de enseñanzaaprendizaje que, por una parte, prioricen la participación activa de los alumnos en este proceso y, por otra, establezcan una relación diferente entre docentes y discentes. Son muchas las acciones de innovación educativa que se están poniendo en práctica en la Universidad española, con el fin de superar los esquemas didácticos tradicionales y lograr una enseñanza de calidad, objetivos fundamentales del proceso de convergencia europea que se está viviendo en la actualidad. El éxito de estas iniciativas, muchas de ellas apoyadas en las denominadas Tecnologías de la Información y de la Comunicación (TIC) depende, por una parte, de la actitud y del compromiso de los docentes con el cambio metodológico y, por otro, del apoyo que se les brinda desde la institución universitaria para satisfacer sus necesidades formativas y facilitar, así, la incorporación de estos nuevos recursos al proceso de enseñanza-aprendizaje (E/A).

Este artículo presenta un estudio de las actitudes de los profesores de la Facultad de Traducción e Interpretación de la Universidad de Valladolid (España) hacia la integración de 
las TIC en su práctica docente, ya que consideramos que la dimensión actitudinal representa un elemento clave para la renovación pedagógica exigida por el Espacio Europeo de Educación Superior (EEES).

PALABRAS CLAVE: Actitudes, EEES, Tecnologías de la Información y de la Comunicación (TIC), innovación docente.

\section{ABSTRACT}

The process of European convergence and harmonization and the EHEA-adaptation plan that our universities are implementing nowadays entail not only a methodological change- that is the introduction of active methodologies that emphasize the role played by students in their own learning process- but also a change in the role assumed by teachers in the teachinglearning process. Spanish universities are currently carrying out many experimental and innovative educational projects which try to overcome traditional methodological limitations in order to guarantee solid and quality educational programs in our higher education institutions. Achieving success in innovative instructional practices-most of them based on ICTs- depends both on university teachers' attitude and commitment to the methodological changes involved and on the institutional and organizational support provided by universities in order to enhance ICT competence development of teachers. That is teachers' attitudes towards ICTs, which in most cases are related to their ICT competence level, play a crucial role in implementing new innovative tools in the teaching-learning process.

This research article shows the attitudes of a group of teachers of the Faculty of Translation and Interpreting (University of Valladolid) to the educational use of ICTs, as we consider that the attitudinal dimension is a key issue in the integration of technology into educational environments.

KEYWORDS: Attitude, Information and Communication Technologies (ICTs), educational innovation.

\section{INTRODUCCIÓN}

En las últimas décadas del siglo XX, las nuevas tecnologías comenzaron a abrirse paso en el aula española, permitiendo al profesor encontrar un complemento para su actividad docente; sin embargo, no ha sido hasta tiempos recientes, y en particular a raíz de la cultura impuesta por la denominada "Sociedad de la Información", cuando se han incorporado de manera contundente a la rutina del docente. Esto se ha hecho sentir en todos los niveles, pero especialmente en el ámbito universitario, inmerso en la actualidad en el proceso de convergencia europea. En este nuevo marco universitario común europeo (Espacio Europeo de Educación Superior, en adelante EEES), el recurso a las Tecnologías de la Información y de la Comunicación (TIC) se presenta ya no solo como una posible preferencia personal del docente, sino como una necesidad impuesta desde fuera.

Como consecuencia de las nuevas directrices europeas, la presencialidad en el aula universitaria se ve reducida de manera notoria, reposando ahora el sistema de enseñanza en nuevos canales comunicativos entre el docente y el discente, y modelos de aprendizaje 
colaborativos, que favorecen el proceso de adquisición y desarrollo de conocimientos y competencias en el alumno. En este marco, las TIC nos permiten desarrollar herramientas metodológicas novedosas y flexibles en el proceso de enseñanza-aprendizaje (E/A), que favorecen la participación activa de los estudiantes y les convierten en los auténticos protagonistas de su aprendizaje.

En este panorama de cambio, el profesor universitario se convierte en parte fundamental del proceso de adaptación al nuevo sistema de enseñanza, siendo el principal responsable del buen uso y aprovechamiento de estos nuevos recursos. Queda fuera de toda duda que las nuevas tecnologías han abierto un sinfín de posibilidades en lo referente a la metodología, pero esta imposición -que implica la superación de los esquemas didácticos tradicionales- puede ser contemplada por muchos de nosotros como un desafío en un momento en el que las reticencias hacia el uso -y previo aprendizaje- de estas tecnologías aún están presentes entre los docentes, siendo un factor de importancia mayor que puede condicionar su aplicación y los resultados consecuentes en el aula.

Esta contribución se centra así en el gran bloque de "factores personales" (Gargallo et al., 2004), dejando a un lado los factores y valoraciones referentes a los ámbitos políticoadministrativos $u$ organizativos. Tomando como objetivo valorar la actitud de los docentes universitarios hacia la integración de las TIC en sus aulas, un grupo de profesores e investigadores de la Facultad de Traducción e Interpretación de la Universidad de Valladolid (España) ha procedido a aplicar un instrumento de valoración que permita aportar un muestrario ejemplificador y descriptivo de esta circunstancia en su centro académico. Esto ayudará a sacar conclusiones ilustrativas de cara a fomentar diversas acciones de innovación educativa que ya se están poniendo en práctica en la Universidad española.

La pertinencia y motivación de esta pequeña aportación reposa en el hecho de que "al analizar la integración de las TIC en los procesos educativos hay que considerar no sólo las argumentaciones racionales sino también las emociones que hay detrás de ellas" (GarcíaValcárcel y Tejedor, 2007), emociones que vienen a aumentar la complejidad de este proceso de integración. En otras palabras, la dimensión actitudinal del docente representa un elemento clave para la renovación pedagógica exigida por el EEES.

Esta práctica forma parte del Proyecto de Innovación Educativa que integra este mismo equipo de profesores y que, titulado "Nuevas herramientas metodológicas basadas en Web 2.0. para la adquisición de competencias en asignaturas de Traducción: una adaptación al EEES", se impone como principal objetivo diseñar estrategias educativas que faciliten la implicación activa de los estudiantes en su proceso de autoaprendizaje y contribuir así a perfilar la revolución metodológica defendida en el denominado proceso de Bolonia.

\section{FUNDAMENTACIÓN TEÓRICA: LAS ACTITUDES}

El concepto de actitud se ha definido tradicionalmente como una disposición a reaccionar favorable o desfavorablemente hacia un objeto, situación o suceso. Las actitudes, conjuntamente con la personalidad, la motivación, las expectativas de cada persona, la experiencia sociocultural o la ansiedad, se engloban dentro de las denominadas variables afectivas de aprendizaje (Skehan, 1989). 
La mayoría de las definiciones de actitudes que encontramos en la literatura se pueden agrupar en tres categorías (Olson y Zanna, 1993): componente afectivo (sentimientos hacia...), componente conductual (acciones o conductas hacia...) y componente cognitivo (pensamientos hacia...).

Por otra parte, las actitudes, al igual que el resto de variables afectivas y como el propio proceso de aprendizaje, no son nunca estáticas, sino que varían y se pueden ver alteradas o modificadas a lo largo del tiempo influenciadas por personas, experiencias y contextos (Herdina y Jessner, 2002; Adegbija, 1994). Este cambio de actitudes se puede ver afectado por varios agentes, como las instituciones, tal y como indica McGuire

Institutions may affect attitudes, not only by their explicit persuasive communications, but also by their structures' determining the stimulus situation to which the person is exposed, the response options made available, the level and type of motivation aroused, and the schedules of reinforcements administered" (1999: 103).

Podemos aseverar, del mismo modo, que las actitudes representan una pieza indispensable en el mundo científico y que cumplen funciones principales, puesto que se pueden medir con un alto grado de fiabilidad y validez, a lo que se añade que son de gran utilidad cuando se trata de predecir el comportamiento. Este papel fundamental es el que nos ha conducido a realizar un estudio sobre las actitudes que reflejan los profesores de la Facultad de Traducción e Interpretación ante las nuevas tecnologías y ante su integración en el aula, que nos permitirá analizar qué factores determinan y definen dichas actitudes, proponer un modelo de análisis y ver cómo podemos hacer uso de esta información para promover una universidad de "digital immigrants" más seguros y motivados en el uso de las nuevas tecnologías con sus estudiantes "digital natives"

\section{METODOLOGÍA}

Tal y como hemos expuesto en la introducción del presente artículo, el objetivo principal de nuestra investigación era analizar las actitudes de los docentes de la Facultad de Traducción e Interpretación de la Universidad de Valladolid (España) ante las Tecnologías de la Información y de la Comunicación y su integración en el aula.

Para ello se contó con la participación voluntaria de 25 profesores de dicha Facultad $(62,5 \%)$, de un total de 40 que conforman la plantilla docente del centro.

\subsection{Instrumentos de medición}

El instrumento de recogida de información es un cuestionario de actitudes (Anexo I), que fue elaborado y puesto a disposición de la comunidad educativa por García-Valcárcel y Tejedor

\footnotetext{
Ambos conceptos fueron introducidos por Marc Prensky (2001) para representar las diferencias generaciones existentes entre los docentes y los discentes actuales en cuanto al dominio de la tecnología digital. Así, los alumnos del siglo XXI son, en palabras del autor, "native speakers of the digital language of computers, video games and the Internet" (ibídem: 1); sin embargo, los docentes, en comparación con ellos, se conciben como "inmigrantes digitales", que han de adaptarse al lenguaje y a las particularidades de este nuevo mundo digital.
} 
$(2007)^{2}$. Se trata de un instrumento de medición válido y fiable, cuyo coeficiente alfa de Cronbach de consistencia interna es 0.961 . Consta de 30 ítems valorados en una escala tipo Likert con un rango de respuesta de entre 1 y 5 (1: muy de acuerdo-MA; 2 : de acuerdo-A; 3 : indiferente-I; 4: desacuerdo-D y 5: muy en desacuerdo-MD). Los ítems que forman parte del cuestionario tienen la intención de medir, de forma indirecta, diferentes actitudes de los sujetos, en este caso del profesorado de la Facultad de Traducción e Interpretación, hacia el objeto considerado, o lo que es lo mismo, las Tecnologías de la Información y de la Comunicación.

Los ítems son claros, valorativos e incluyen una única idea; sin embargo, también es necesario hacer constar que son de naturaleza variada; por este motivo, a la hora de medir las actitudes, decidimos agrupar los ítems en cuatro categorías o dimensiones (Tabla 1), que pretenden hacer un recorrido por aquellos aspectos que conforman el marco actitudinal del profesorado en relación con las TIC. Estas dimensiones responden a los siguientes aspectos:

En primer lugar, la actitud del profesorado en cuanto al cambio metodológico impuesto por las TIC: tal y como hemos afirmado previamente, el Espacio Europeo de Educación Superior supone un reto en cuanto a cambio en la concepción de la docencia y, en consecuencia, en las metodologías a emplear. Las TIC se conciben como herramientas que nos ayudan a definir ese cambio, por lo que, en primer lugar, queríamos medir la actitud del profesorado ante ese cambio general. Por este motivo, algunos ítems se agruparon bajo la dimensión que denominamos "Posición personal general frente a las TIC aplicadas a la educación".

En segundo lugar y en relación con el punto anterior, nuestra siguiente cuestión era medir la actitud del profesorado en aspectos más concretos de su práctica docente; en otras palabras, valorar su actitud con respecto a la aplicación de estas herramientas metodológicas a las asignaturas que imparte. Esta dimensión, a la que denominamos "Posición frente al uso educativo que hago de las TIC como docente: aplicación de las TIC a mi docencia en concreto", agrupa aquellos ítems que miden si el profesor está a favor del cambio que supone el EEES, pero muestra una actitud diferente (positiva o negativa) ante la implantación de ese cambio en sus asignaturas. En muchos casos, esa actitud puede ser diferente y queríamos conocer si esta hipótesis se materializaba en muestro profesorado.

En tercer lugar, decidimos medir la actitud del profesorado en cuanto a la repercusión de las nuevas herramientas metodológicas en el proceso de aprendizaje, es decir, si los docentes consideran que las TIC como reto (positivo o negativo) suponen para el discente una mejora en el proceso de E/A. Los ítems correspondientes a este aspecto los incluimos en una dimensión denominada "Valoraciones relativas a las repercusiones del uso docente de las TIC en el proceso de aprendizaje".

\footnotetext{
Para obtener información más detallada sobre el proceso de construcción y validación de dicho cuestionario, consúltese GarcíaValcárcel y Tejedor (2007).
} 


\begin{tabular}{|c|c|}
\hline DIMENSIONES & ÍTEMS \\
\hline $\begin{array}{l}\text { 1. Posición personal general frente a las } \\
\text { TIC aplicadas a la educación }\end{array}$ & $3,4,7,8,9,11,12,16,19,23,26$ у 28. \\
\hline $\begin{array}{l}\text { 2. Posición frente al uso educativo que } \\
\text { hago de las TIC como docente: aplicación } \\
\text { de las TIC a mi docencia en concreto. }\end{array}$ & $2,5,10,13,14,15,20,21,27$ у 29 \\
\hline $\begin{array}{l}\text { 3. Valoraciones relativas a las } \\
\text { repercusiones del uso docente de las TIC } \\
\text { en el proceso de aprendizaje } \\
\text { 4. Percepciones relacionadas con las } \\
\text { necesidades implicadas por las TIC: } \\
\text { infraestructuras }\end{array}$ & $1,17,22,24,25$ у 30 \\
\hline
\end{tabular}

Tabla 1. Clasificación de los ítems del cuestionario aplicado.

Por último, agrupamos los ítems que no tenían nada que ver con los aspectos o factores personales, como en el caso de las tres dimensiones anteriores, sino con aspectos organizativos y administrativos. Esta última dimensión, que denominamos "Percepciones relacionadas con las necesidades implicadas por las TIC: infraestructuras", pretende medir la importancia concedida por los docentes a los medios o infraestructuras disponibles en los centros para el desarrollo de una práctica metodológica con apoyo tecnológico.

Con esta agrupación de los 30 ítems en diferentes dimensiones, hemos pretendido cubrir totalmente el marco actitudinal del profesorado desde lo más genérico a lo más específico, incluyendo aspectos organizativos y administrativos.

\subsection{Procedimiento y análisis de datos}

El cuestionario de actitudes elaborado fue realizado con la herramienta gratuita Google-Docs (forms $)^{3}$, que permite exportar los datos a un archivo Excel, lo que facilita en gran medida su posterior análisis. El hipervínculo a este cuestionario online fue enviado a todo el profesorado de la facultad mediante un correo electrónico, en el que se expusieron los objetivos de la investigación y se les animó a participar en el mismo.

Debido a la naturaleza del estudio, el análisis se limitó a la realización de la estadística descriptiva de los ítems, por una parte, y de cada una de las dimensiones de ítems en conjunto, por otra, mediante el programa SPSS 15 (Statistical Package for the Social Sciences). Este instrumento de análisis estadístico nos permite medir, no solo las respuestas individuales de cada ítem, sino también obtener una media de las respuestas de cada una de las dimensiones en las que agrupamos los ítems; esta segunda operación nos permite extraer conclusiones de una forma mucho más clara.

\footnotetext{
$3 \quad$ El cuestionario aparece publicado en la siguiente dirección: https://spreadsheets.google.com/viewform?formkey=dGM2SDAzMTFCREdyUDVxb25nYVJEVXc6MA
} 


\section{RESULTADOS}

Los resultados de la investigación que a continuación exponemos son de carácter descriptivo y están estructurados en torno a las diferentes dimensiones de ítems en las que hemos dividido el cuestionario utilizado, aunque también destacaremos los resultados obtenidos en algunos ítems en concreto, que consideramos de especial relevancia para la consecución de los objetivos de nuestro trabajo.

Con el fin de facilitar la interpretación de los datos, las respuestas a las ítems se han colapsado en 3 categorías: en desacuerdo (muy en desacuerdo y en desacuerdo), indiferente y de acuerdo (muy de acuerdo y de acuerdo).

\section{A. Posición personal general frente a las TIC aplicadas a la educación}

El 92\% (48\%, MA y 44\%, A) de los profesores que han cumplimentado la encuesta consideran que las TIC son muy importantes para la enseñanza en el momento actual y todos ellos opinan que los docentes desempeñan un papel muy importante en el proceso de integración de las mismas. Asimismo, todos los profesores encuestados consideran necesario realizar un esfuerzo de actualización (48\%, MD y $52 \%$, A) para sacar el máximo partido a todas las potencialidades que estas herramientas tecnológicas nos brindan.

Asimismo, el $88 \%$ ( $52 \%$, D y $36 \%, M D)$, en contraposición al $12 \%$ que se muestra indiferente, opina que las TIC no solo sirven para adornar la docencia y el $92 \%$ de ellos $(20 \%$, MD y $72 \%$, D) - en comparación con el $8 \%$, que se muestra indiferente ante este ítem- estima que estas herramientas no pueden considerarse como elementos que entorpecen el proceso de $E / A$, sino como herramientas que ayudan a definirlo.

Por otra parte, podemos vislumbrar en los resultados un alto grado de motivación en los docentes de nuestro centro ante la integración de las TIC en los actos formativos, ya que todos ellos $(36 \%$, MA y $64 \%, A)$ están dispuestos a aprender las posibilidades que estos recursos brindan al mundo de la enseñanza y no se encuentran preocupados (24\%, MD y $44 \%$, D) por la integración masiva de estas herramientas tecnológicas en las aulas, como consecuencia de lo que algunos han denominado la "tercera revolución educativa" (Esteve, 2003).

En general, podríamos afirmar que los docentes encuestados consideran que el uso de las TIC les ayudará a mejorar su función docente ( $29 \%$ MA y $50 \%$ A), tal y como podemos observar en la figura 1. 


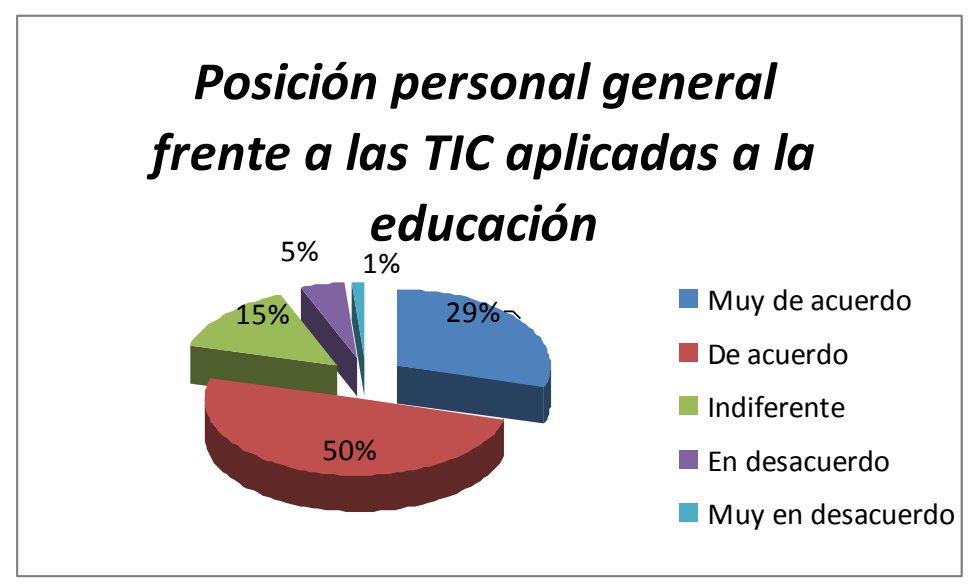

Figura 1. Media de las preguntas correspondientes a la dimensión "Posición personal general frente a las TIC aplicadas a la Educación".

La media de las respuestas de esta primera dimensión se sitúa en 2,17 , es decir, entre el valor "de acuerdo" y el de "indiferente".

B. Posición frente al uso educativo que hago de las TIC como docente: aplicación de las TIC a mi docencia en concreto

En relación con esta dimensión, cabría decir que el profesorado que ha rellenado la encuesta señala la gran importancia que tiene la integración de las TIC en su práctica docente (la media de los ítems de esta dimensión es de 1,89); en este sentido, el $80 \%{ }^{4}$ de los encuestados considera que sus prácticas docentes van a mejorar considerablemente si integran las TIC en su prácticas metodológicas y el $88 \%$ de ellos estima conveniente introducirlas en sus clases, en contraposición al $4 \%$ que se muestra indiferente y al $8 \%$ que no lo considera pertinente. En la figura 2 se representan los valores medios de esta dimensión, formada por las respuestas a los ítems 2,5,10,13, 14, 15, 20, 21, 27 y 29.

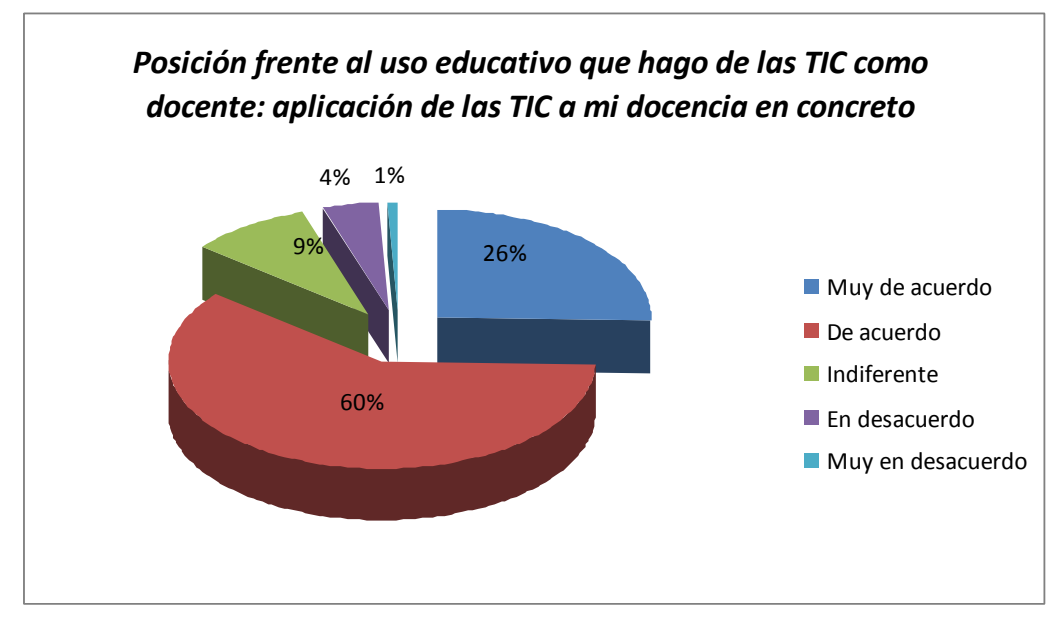

Figura 2. Media de las preguntas correspondientes a la dimensión "Posición frente al uso educativo que hago de las TIC como docente: aplicación de las TIC a mi docencia en concreto".

\footnotetext{
$4 \quad$ El 16\% de los profesores responde" muy en desacuerdo" (MD) al ítem negativo "mis prácticas docentes no van a mejorar por el uso de las TIC" y el 64\% se muestra "en desacuerdo" (D).
} 
Resulta significativo en esta dimensión el hecho de que a casi todos los profesores (96\%: $35 \%$, MA y $60 \%, A$ ) les resulte positivo ir integrando las TIC en las materias que imparten con el fin de enriquecerlas y que el $72 \%$ (24\%, MA y $48 \%$, A) confíe plenamente en estas herramientas como motor de cambio metodológico hacia modelos de E/A más útiles $\mathrm{y}$ flexibles para los discentes.

Al mismo tiempo, los datos reflejan que los profesores se encuentran motivados, ya que reconocen que, aunque la integración de las TIC puede suponer un esfuerzo adicional a su carga de trabajo, es un hecho necesario en la Sociedad de la Información. La figura 3 representa cómo al $96 \%$ de los docentes $(28 \%$, MA y $68 \%$, A) les parece conveniente esforzarse por integrar las TIC en el curriculum de su asignatura, hecho que pone de manifiesto la confianza depositada en estas herramientas en el ámbito educativo.

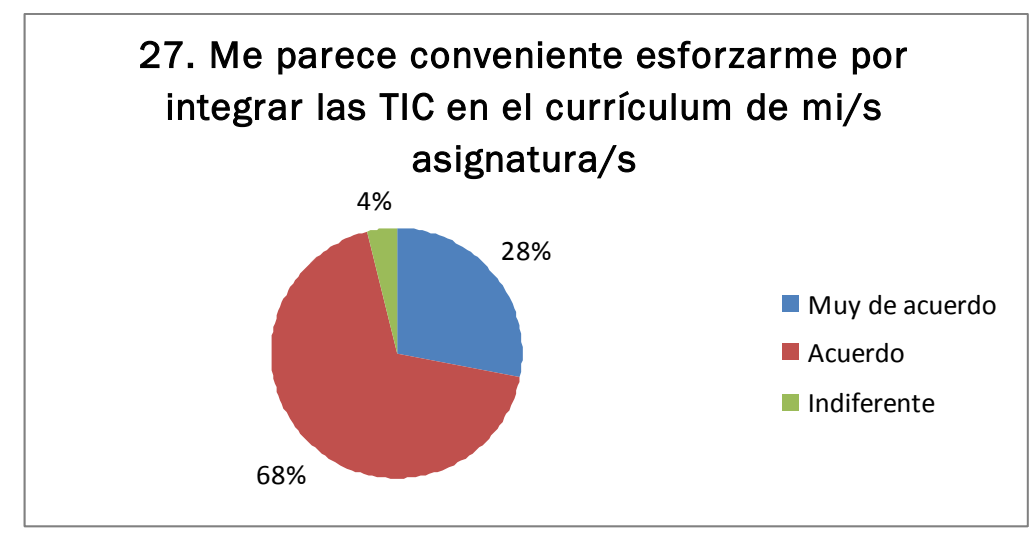

Figura 3. Respuestas al item 27 ("Me parece conveniente esforzarme por integrar las TIC en el currículum de $\mathrm{mi} / \mathrm{s}$ asignatura/s").

\section{Valoraciones relativas a las repercusiones del uso docente de las TIC en el proceso de aprendizaje}

Con esta escala de ítems pretendíamos analizar lo que opinaban los docentes con respecto a las posibilidades que ofrecen las Tecnologías de la Información y de la Comunicación en el proceso de aprendizaje del discente. La media de las respuestas fue de 1,96, luego los profesores se muestran entre "muy de acuerdo" y "de acuerdo" con los ítems de esta dimensión (veáse Figura 4):

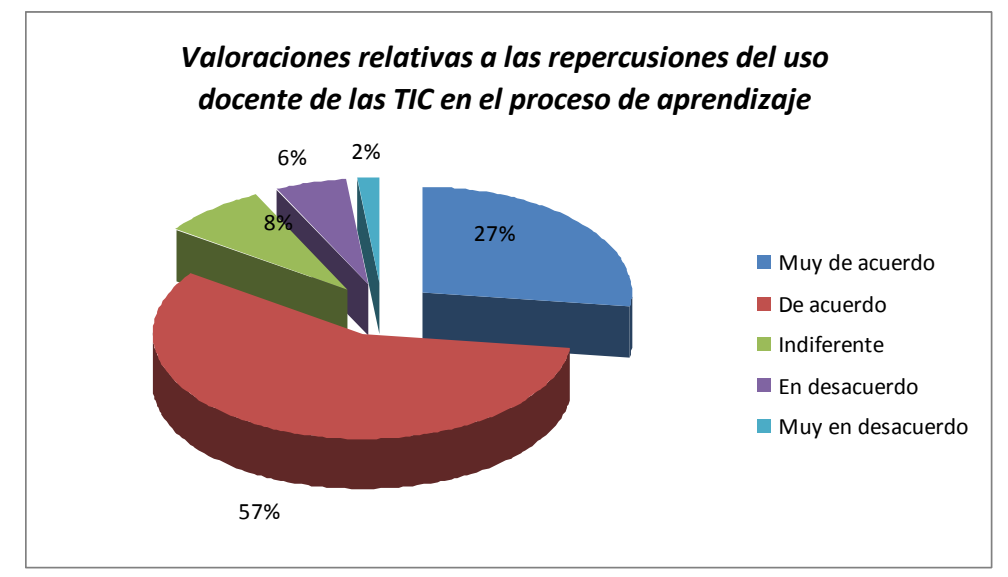

Figura 4. Media de las preguntas correspondientes a la dimensión "Valoraciones relativas a las repercusiones del uso docente de las TIC en el proceso de aprendizaje". 
El $84 \%$ de los docentes que han cumplimentado el cuestionario no están de acuerdo (12\%, MD y $72 \%$, D) con el ítem "la utilización de las TIC no permite desarrollar un aprendizaje significativo para los estudiantes", en contraposición con el $12 \%$ que se muestra indiferente ante este ítem. A su vez, el $76 \%(20 \%$, MA y $56 \%$, A) considera que se deberán utilizar las nuevas herramientas tecnológicas para ayudar a los alumnos durante su proceso formativo, ya que les permite ejercitarse en la adquisición de algunas destrezas intelectuales básicas y les facilitan la construcción de su propio conocimiento.

Como podemos observar en la figura 5, el $92 \%$ de los encuestados (40\%, MD y $52 \%$, D) no está de acuerdo con el ítem "las TIC no favorecen el aprendizaje activo por parte de los alumnos" y el $96 \%$ de ellos (44\%, MA y 52\%, A) estima que la utilización de estas herramientas en algunas actividades suponen un buen modo de aprender (figura 6).

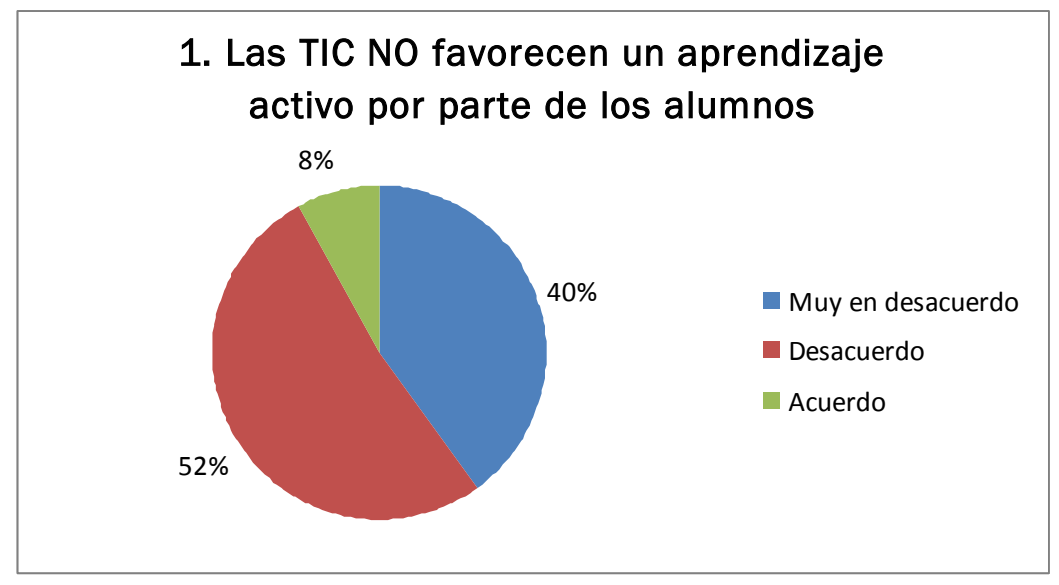

Figura 5. Respuestas al ítem 1 ("Las TIC no favorecen un aprendizaje activo por parte de los alumnos").

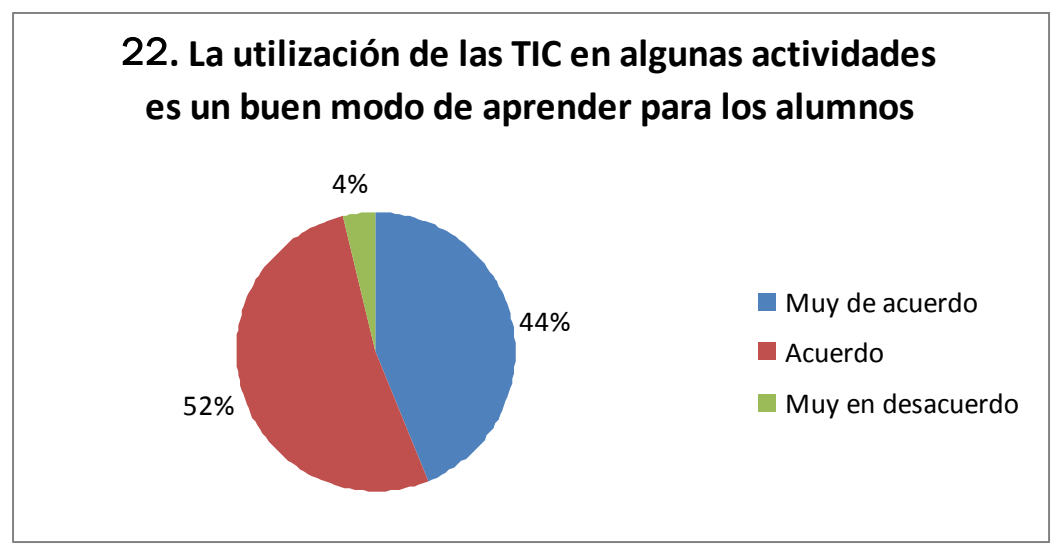

Figura 6. Respuestas al ítem 22 ("La utilización de las TIC en algunas actividades es un buen modo de aprender para los alumnos).

Por otra parte, el $68 \%$ de los profesores ( $32 \%$, MA y $36 \%$, A), en contraposición al $28 \%$ que se muestra indiferente, reconoce las potencialidades de las TIC como herramientas flexibles de comunicación que mejoran la interacción entre los participantes en el proceso formativo.

\section{Percepciones relacionadas con las necesidades implicadas por las TIC: infraestructuras}

Por último, en la dimensión que analiza la importancia concedida por los docentes a las infraestructuras necesarias para incorporar la tecnología a las prácticas docentes, cabría 
decir que la mayor parte de ellos ( $80 \%$ : $28 \%$, MA y $52 \%$, A), en oposición al $24 \%$ que permanece en una posición neutral, considera que se debería impulsar la mejora infraestructural en los centros en materia de TIC, puesto que su integración depende no solo de la competencia que tengan los docentes, sino también de los recursos de los que se dispone para llevar a cabo dicha integración. Asimismo, al $68 \%$ de los docentes encuestados (48\%, MA y $20 \%$ A) le encantaría trabajar en un centro con más recursos tecnológicos, en contraposición al $24 \%$ que se manifiesta indiferente y al $8 \%$, que se muestra en desacuerdo.

\section{DISCUSIÓN Y CONCLUSIONES}

La integración de las TIC en el mundo educativo no solo depende de su calidad técnica y de sus posibilidades pedagógicas, sino también del enfoque y de la metodología docente de la que formen parte. Por este motivo, los conocimientos, percepciones y actitudes que tengan los profesores con respecto a los medios se convertirán en factores determinantes a la hora de integrarlos en los procesos formativos. Es decir, "las emociones van a determinar el grado de integración de las TIC en las prácticas educativas" (García-Valcárcel y Tejedor, 2007). Tomando como punto de partida esta idea, hemos llevado a cabo un estudio descriptivo cuyo principal objetivo era conocer la actitud general de los docentes de nuestra facultad ante las TIC aplicadas a la educación.

En términos globales, los resultados de los cuestionarios muestran una actitud general positiva ante este constructor por parte de los docentes de nuestro centro, dentro del proceso de renovación de las concepciones y prácticas docentes exigido a las universidades en el marco de la sociedad tecnológica actual.

No cabe duda de que la implantación de los nuevos programas educativos derivados del proceso de Bolonia y la utilización de recursos y medios de E/A innovadores plantean no solo una nueva configuración del proceso didáctico y metodológico, sino también el desempeño de nuevos roles docentes, roles para los que han de estar preparados, ya que, "los profesores son la clave del cambio" (Orellana, Almerich et al., 2004). En este sentido, el análisis de los resultados pone de manifiesto que los profesores de la Facultad de Traducción e Interpretación de la Universidad de Valladolid reconocen el destacado papel que juegan como docentes en la integración de las TIC en el aula. De la misma manera, coinciden en subrayar la relevancia que estas tienen en el proceso de E/A.

Por otra parte, los datos derivados de las encuestas muestran que el profesorado de nuestro centro es consciente de que las TIC son una realidad que han de conocer y utilizar si no quieren quedar desfasados. Las conciben como un reto que están abordando poco a poco, puesto que reconocen las potencialidades que estas brindan al proceso formativo. Además, como cabía de esperar, se augura un buen futuro, puesto que todos ellos se han mostrado dispuestos a realizar un esfuerzo de actualización para sacar el máximo partido a todas las potencialidades que estas herramientas tecnológicas nos brindan. Por otra parte, será necesario impulsar la mejora infraestructural en materia de TIC en los centros, ya que su integración va ligada también a los recursos tecnológicos de que disponga el profesorado en su trabajo diario.

Somos conscientes de que el número de participantes en la muestra no es muy elevado; sin embargo, lo consideramos representativo (62,5\% de los profesores de la Facultad) para los 
objetivos perseguidos en nuestro trabajo. En futuras investigaciones en este campo, resultaría necesario aumentar el número de sujetos participantes en la muestra y analizar también otras variables tales como el género, la edad, la categoría profesional, el grado de competencia en TIC o la materia que se imparte, con el fin de valorar cómo afectan estos parámetros a la actitud de los docentes ante las herramientas tecnológicas y su integración en las aulas.

Nuestros resultados son coherentes con los obtenidos en investigaciones anteriores tales como Orellana, Almerich et al. (2004), Gargallo, Suárez et al (2004) o Fernández, Hinojo y Aznar (2002), hecho que nos hace reflexionar sobre la necesidad de trabajar las actitudes de los docentes ante las TIC además de sus competencias y destrezas en esa materia. No olvidemos que la opinión de los docentes sobre el potencial didáctico de las TIC va a condicionar el uso que hagan de estas herramientas en su práctica docente. En este sentido, resulta crucial conocer las actitudes que expresan los profesores en referencia a su empleo en el proceso de E/A.

\section{REFERENCIAS BIBLIOGRÁFICAS}

ADEGBIJA, E. (1994). Language Attitudes in Sub-Saharan Africa. Clevedon: Multilingual Matters.

BENITO, A., \& CRUZ, A. (Coords.) (2005). Nuevas claves para la Docencia Universitaria en el Espacio Europeo de Educación Superior. Madrid: Narcea.

ESCANDELL BERMÚDEZ, M.O., RODRÍGUEZ MARTíN, A., \& CARDONA HERNÁNDEZ, G. (2005). Convergencia europea y profesorado. Hacia un nuevo perfil para el aprendizaje flexible. En Revista Electrónica Interuniversitaria de Formación del Profesorado (REIFOP), 20, Vol. 8 (5), (pp. 17-21).

ESTEVE ZARAZAGA, J.M. (2003). La tercera revolución educativa: la educación en la Sociedad del Conocimiento. Barcelona: Paidós Ibérica.

FERNÁNDEZ MARTÍN, F., HINOJO LUCENA, F., \& AZNAR DÍAZ, I. (2002). Las actitudes de los docentes hacia la formación en Tecnologías de la Información y Comunicación (TIC) aplicadas a la Educación. En Contextos Educativos, 5 (pp. 253-270).

GARCÍA-VALCÁRCEL MUÑOZ-REPISO, A., \& TEJEDOR TEJEDOR, F.J. (2007). Estudio de las actitudes del profesorado universitario hacia la integración de las TIC en su práctica docente. Extraído de 10을 Congreso Iberoamericano EDUTEC 2007, Buenos Aires (Argentina), 23-25 Octubre. Disponible en http://gredos.usal.es/jspui/handle/10366/18450. [Fecha de consulta: 12/08/2009].

GARGALLO, B., SUÁREZ, J., BELLOCH, C. et al. (2004). Perfiles actitudinales de los profesores ante las TIC e incidencia de las actitudes sobre su uso. Conferencia presentada en Virtual Educa Barcelona 2004. Extraído de http://espacio.uned.es/fez/view.php?pid=bibliuned:19584. [Fecha de consulta: 20/07/2009].

HERDINA, P. \& JESSNER, U. (2002). A Dynamic Model of Multilingualism: Perspectives of Change in Psycholinguistics. Clevedon: Multilingual Matters. 
LÓPEZ-CASTEDO, A. \& FERNÁNDEZ EYRE, L. (2007). Actitud de los universitarios ante las Nuevas Tecnologías de la Información. En Revista Galego-Portuguesa de Psicoloxía e Educación, 15 (2), (pp. 105-113).

MCGUIRE, W.J. (1999). Constructing Social Psychology. Creative and Critical Processes. Cambridge: Cambridge University Press.

OLSON, J.M. \& ZANNA, M.P. (1993). Attitudes and attitude change. En Annual Review Psychology, 44, (pp. 117-154).

ORELLANA, N., ALMERICH, G., et al. (2004). La actitud del profesorado ante las TIC: un aspecto clave para la integración. Conferencia presentada en Virtual Educa Barcelona 2004. Extraído de http://e-spacio.uned.es/fez/view.php?pid=bibliuned:19586. [Fecha de consulta: 20/07/2009].

PRENSKY, M. (2001). Digital Natives, Digital Immigrants. En On the Horizon, Vol. 9 (5), October 2001. MCB University Press.

SKEHAN, P. (1989). Individual Differences in Second-Language Learning. Londres: Edward Arnold.

TEJEDOR, F.J., GARCÍA-VALCÁRCEL, A., \& PRADA, S. (2009). Medida de actitudes del profesorado universitario hacia la integración de las TIC. En Comunicar, 33 (XVII), (pp. 115124).

\section{Para citar este artículo:}

ÁlVAREZ, S.; CUÉlLAR, C.; LÓPEZ, B.; ADRADA, C.; ANGUIANO, R.; BUENO, A.; COMAS, I.; GÓMEZ, S. (2011) «Actitudes de los profesores ante la información de las TIC en la práctica docente. Estudio de un grupo de la Universidad de Valladolid.» [artículo en línea]. EDUTEC, Revista Electrónica de Tecnología Educativa. Núm. 35 / Marzo 2011. [Fecha de consulta: $\mathrm{dd} / \mathrm{mm} / \mathrm{aa}]$. http://edutec.rediris.es/revelec2/revelec35/

ISSN 1135-9250. 
Solicitamos tu opinión en relación con el contenido de las frases que aparecen a continuación, en términos de acuerdo/desacuerdo. Señala, por favor, con una X la respuesta que mejor se ajusta a tu opinión. iMUCHAS GRACIAS POR TU COLABORACIÓN!

1. Las TIC NO favorecen un aprendizaje activo por parte de los alumnos *

Muy en desacuerdo

Desacuerdo

Indiferente

C Acuerdo

Muy de acuerdo

2. No considero conveniente introducir las TIC en mis clases*

Muy en desacuerdo

Desacuerdo

Indiferente

C Acuerdo

Muy de acuerdo

3. Considero que las TIC son muy importantes para la enseñanza en el momento actual

*
Muy en desacuerdo
Desacuerdo
Indiferente
C Acuerdo
Muy de acuerdo

4. Los profesores tenemos que hacer un esfuerzo de actualización para aprovechar las posibilidades didácticas de las TIC *
Muy en desacuerdo
Desacuerdo
Indiferente
Acuerdo
Muy de acuerdo

5. Me parece positivo ir integrando progresivamente las TIC en mi materia *

Muy en desacuerdo

C Desacuerdo 
Indiferente

Acuerdo

Muy de acuerdo

6. Me encantaría trabajar en un centro que contara con más recursos tecnológicos *

Muy en desacuerdo

Desacuerdo

Indiferente

C Acuerdo

Muy de acuerdo

7. Me siento a gusto usando una metodología que prescinde de la moda de las TIC *

Muy en desacuerdo

Desacuerdo

Indiferente

Acuerdo

Muy de acuerdo

8. Las TIC sólo sirven para adornar la docencia *

Muy en desacuerdo

Desacuerdo

Indiferente

C Acuerdo

Muy de acuerdo

9. Las TIC en la docencia son entorpecedoras *

Muy en desacuerdo

Desacuerdo

Indiferente

C Acuerdo

Muy de acuerdo

10. Mis clases han mejorado desde que uso las TIC *

Muy en desacuerdo

Desacuerdo

Indiferente

C Acuerdo

Muy de acuerdo

11. Las TIC deberían ser utilizadas por todos los profesores en las distintas materias * 


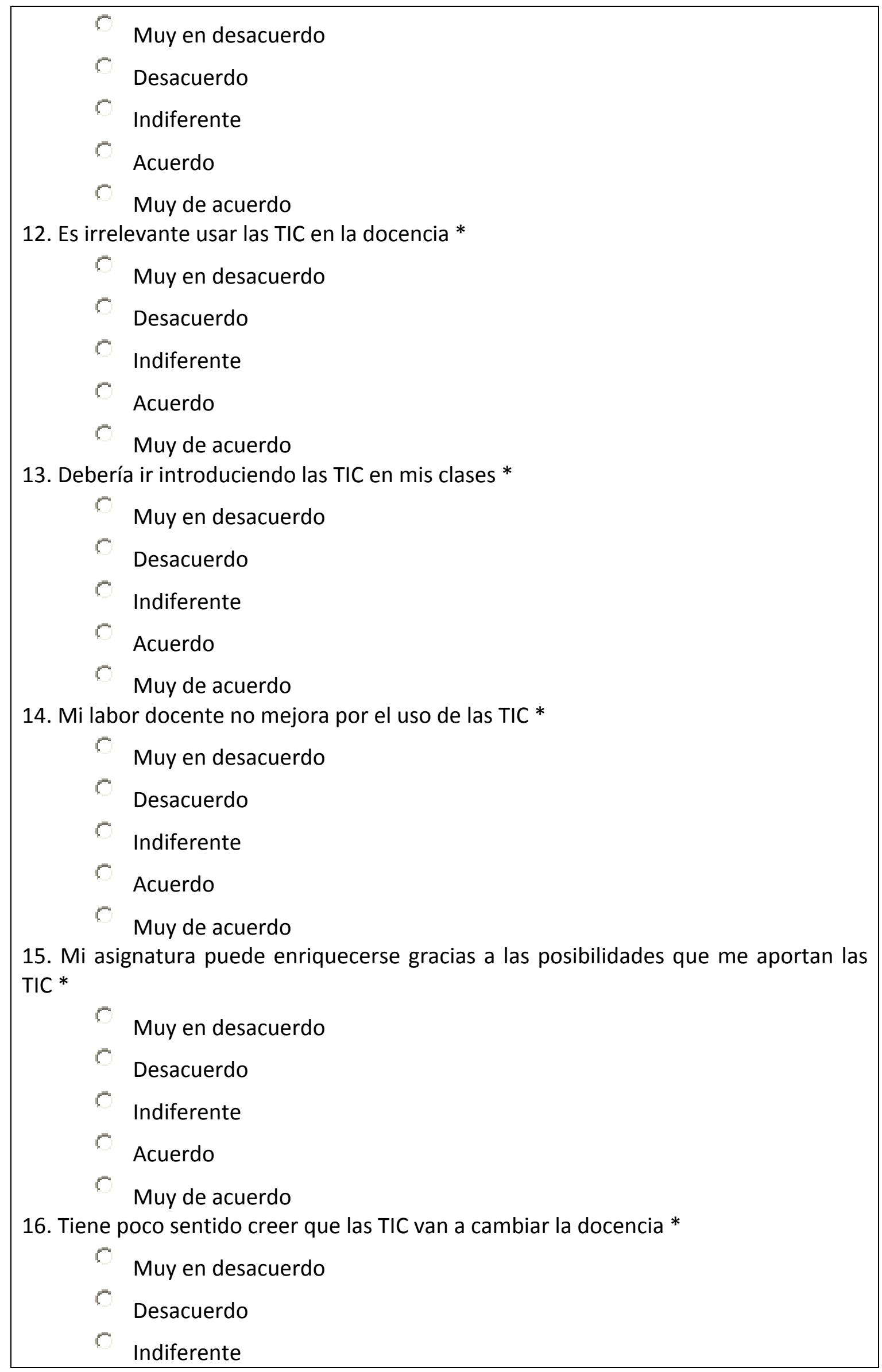




\section{Acuerdo \\ Muy de acuerdo}

17. Las TIC no permiten a los alumnos ejercitarse en la adquisición de algunas destrezas intelectuales básicas *

Muy en desacuerdo

Desacuerdo

Indiferente

C Acuerdo

Muy de acuerdo

18. Debería premiarse la mejora de las infraestructuras actuales en TIC *

Muy en desacuerdo

Desacuerdo

Indiferente

C Acuerdo

C. Muy de acuerdo

19. Estoy dispuesto a aprender las posibilidades de las TIC en la enseñanza *

Muy en desacuerdo

Desacuerdo

Indiferente

C. Acuerdo

C Muy de acuerdo

20. No me parece conveniente para mí introducir las TIC en la docencia *

Muy en desacuerdo

Desacuerdo

Indiferente

C. Acuerdo

Muy de acuerdo

21. Mis prácticas docentes no van a mejorar por el uso de las TIC *

Muy en desacuerdo

Desacuerdo

Indiferente

C Acuerdo

C Muy de acuerdo

22. La utilización de las TIC en algunas actividades es un buen modo de aprender para los alumnos * 


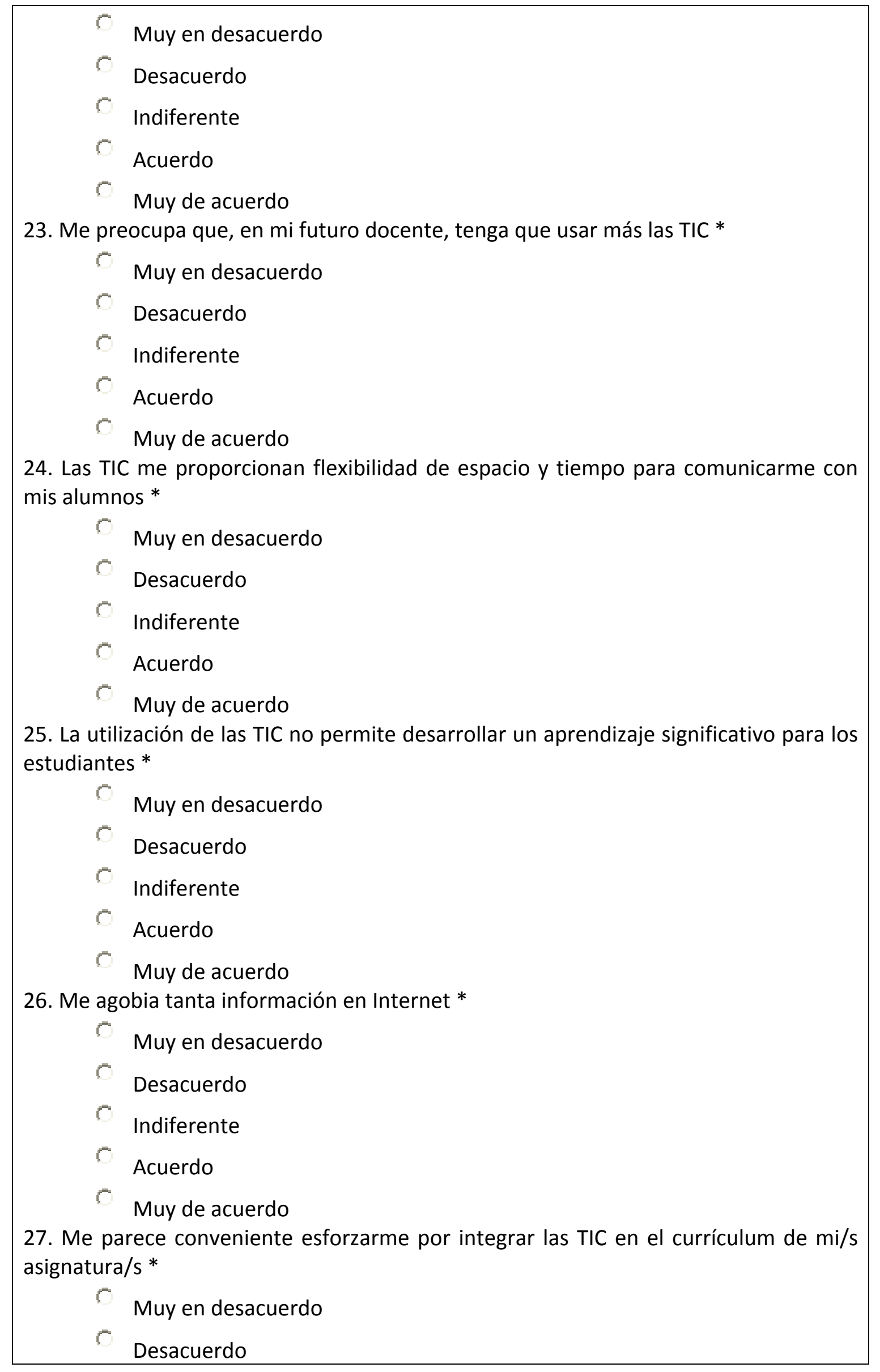




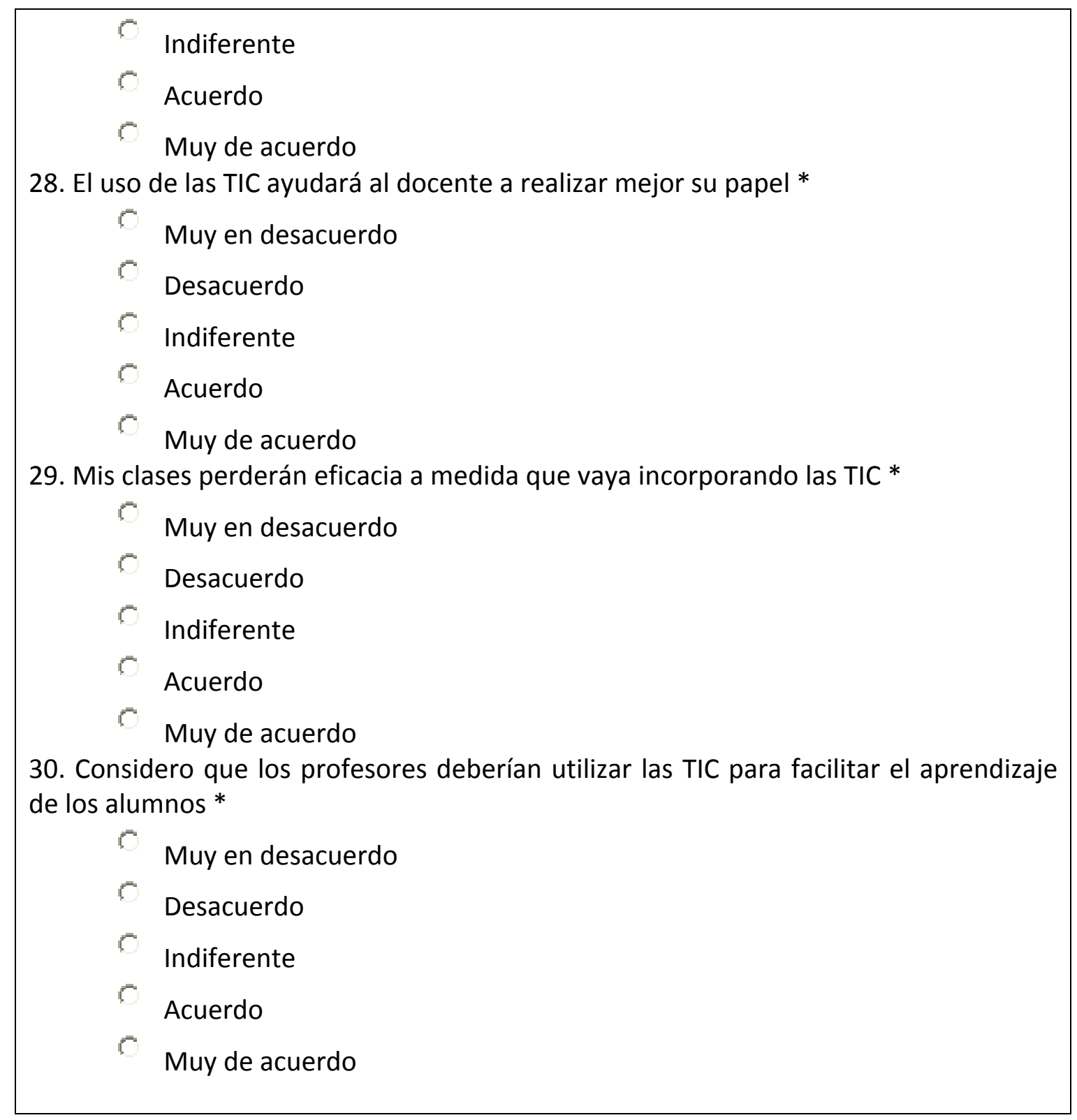

